\title{
Periventricular Leukomalacia
}

\author{
Dr. B.N.Chander ${ }^{1}$, Dr. M. Rajya lakshmi ${ }^{2}$ \\ Professor and $\mathrm{HOD}^{l}$, Post graduate in radio-diagnosis ${ }^{2}$. \\ Department of Radio diagnosis, Dr.Pinnamaneni Siddhartha Institute of Medical Sciences and Research \\ Foundation. Chinnaavutapalli, Andhra Pradesh, INDIA.
}

\begin{abstract}
White matter injury of prematurity (WMIP) was formerly called as Periventricular leukomalacia $(P V L)$ even though it effects gray matter. It represents end stage of WMIP. This article emphasizes role of Neurosonogram in detection \& grading of periventricular leukomalacia and its drawbacks.
\end{abstract}

Keywords: Periventricular leukomalacia (PVL), Neurosonogram, Grading.

\section{Introduction}

Periventricular leukomalacia (PVL) is a white matter injury of prematurity that affects the periventricular zones resulting in cavitations and cyst formation. It is seen in mild to moderate asphyxia in preterm neonates.

\section{Case Report}

A 21 day old preterm baby delivered at 29 weeks of gestation with Apgar score of $5 \& 6$ at 1 minute \& at 5 minutes. Clinically the child had bradycardia \& poor respiratory effort. Neurosonogram was performed on $21^{\text {st }}$ day which revealed periventricular cystic areas \& hyper-echogenic changes. Periventricular region shows cavitations \& cyst formation, anterior \& lateral to frontal horns and lateral \& superior to occipital horns with sub ependymal hemorrhage \& mild hydrocephalus (figure $2 \mathrm{a}, \mathrm{b}$ ) which are characteristic features of Periventricular leukomalacia.

\section{Discussion}

Periventricular leukomalacia (PVL) is a white matter injury of prematurity that affects the periventricular zones and results in cavitations and periventricular cyst formation. It is seen in mild to moderate asphyxia in preterm neonates.

Epidemiology

It occurs most commonly in premature infants born at less than 33 weeks gestation and less than 1500 g birth weight. Pathology

Pre-oligodendrocytes are more vulnerable to hypoxia than matured ones. These are affected due to ischemia and hypoxia resulting from impaired perfusion at the watershed areas, which in premature infants are located in a periventricular location. Motor \& visual pathways are most commonly involved. Lower extremities are more involved than upper extremities due to location of cortico-spinal tracts of lower extremity near the periventricular regions ${ }^{[1,3]}$ (Fig.1) .

Imaging

\section{Neurosonogram}

Cranial ultrasound provides a convenient, non invasive, relatively low-cost screening examination of the hemodynamically unstable neonate at the bedside. Sonography is sensitive for the detection of hemorrhage, periventricular leukomalacia and hydrocephalus. On ultrasound, hyper echoic areas are firstly identified in a distinctive fashion in the periventricular area, more often at the peritrigonal area and in an area anterior and lateral to the frontal \& occipital horns.

These are watershed areas that are sensitive to ischemic injury. Follow-up scans in the more severely affected patients may reveal the development of cysts in these areas, known as cystic Periventricular leukomalacia.

Grading of periventricular leukomalacia based on sonographic appearances ${ }^{[2]}$ :

- Grade 1: Areas of increased periventricular echogenicity without any cyst formation persisting for more than 7 days

- Grade 2: Echogenicity has resolved into small periventricular cysts. 
- Grade 3: Areas of increased periventricular echogenicity that develops into extensive periventricular cysts in the occipital and fronto-parietal region.

- Grade 4 : Areas of increased periventricular echogenicity in the deep white matter developing into extensive subcortical cysts

\section{Neurosonogram Drawbacks:}

- The findings are operator dependent and considerable intraobserver and interobserver variation can occur.

- Ultrasonography is not good for assessing the exact grade of the PVL. It cannot be used to assess the cortical region and the structures of the posterior fossa.

- Studies have shown that MRI is superior to Ultrasonography to in assessing the exact extent and severity of PVL.

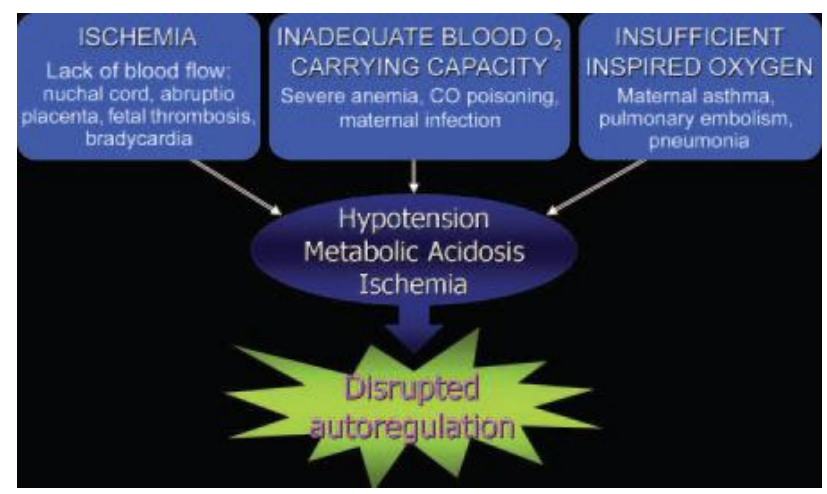

Figure 1. Diagram summarizes the causes of HIE (Hypoxic-Ischemic Encephalopathy). Courtesy: RadioGraphics 2006; 26:S159-S172

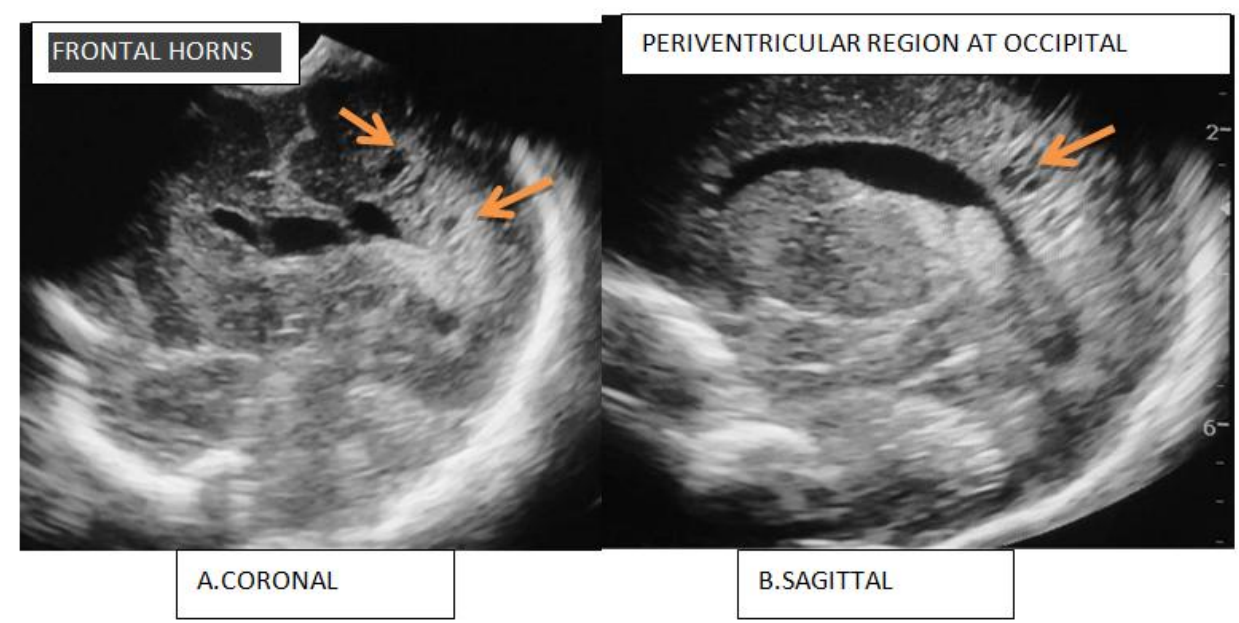

Figure 2 A, B shows periventricular cystic changes at frontal and occipital horns (arrows).

Table 1: Effective times for imaging techniques in perinatal hypoxic - ischemic injury ${ }^{[1]}$.

\begin{tabular}{|l|l|l|}
\hline \multicolumn{1}{|c|}{ TECNIQUE } & FINDINGS & TIMING \\
\hline Ultrasound & Increased echogenicity & $2-10$ Days \\
\hline CT & Low attenuation & $1-7$ Days \\
\hline MRS & Increased lactate, & $1-15$ Days \\
& Decreased NAA & After $3^{\text {rd }}$ day \\
\hline DWI & Reduced diffusion & $1-5$ Days \\
\hline Anatomic MRI & T2 prolongation & 24 hrs \\
\hline T1 shortening & & $2-3$ Days to months \\
\hline T2 shortening & & $6-7$ Days to months \\
\hline
\end{tabular}

- $\quad$ CT - Computed tomography, MRS - Magnetic resonance spectroscopy, DWI - Diffusion weighted imaging, MRI - Magnetic resonance imaging.

Courtesy :Barkovich AJ. ,Pediatric neuroimaging. 4th ed. 


\section{Conclusion}

Neurosonogram is a cheap, easily available, less time taking modality for detecting periventricular leukomalacic changes and follow up.

\section{References}

[1] Barkovich AJ. Brain and spine injuries in infancy and childhood. In: Barkovich AJ, ed. Pediatric neuroimaging. 4th ed. Philadelphia, Pa: LippincottWilliams \& Wilkins, 2005; 190-290.

[2] Dr Yuranga Weerakkody and Dr Bruno Di Muzio et al, Radiopedia.org.

[3] Christine P. Chao, MD et al. Neonatal Hypoxic-Ischemic Encephalopathy:Multimodality Imaging Findings , RadioGraphics 2006; 26:S159-S172. 